\title{
ON THE CONVEX SOLUTION OF A CERTAIN FUNCTIONAL EQUATION ${ }^{1}$
}

\author{
H. P. THIELMAN
}

The object of this paper is to give a generalization of a result obtained in a recent article by Mayer. ${ }^{2}$ His result is a particular case (for $a=1, p=1, K=0$ ) of the following theorem.

THEOREM 1. The only function which is convex for all $x>K \geqq 0$, and satisfies the functional equation

$$
1 / f(x+a)=x^{p} f(x), \quad x>0, a>0, p>0,
$$

is

$$
F(x)=\left[\frac{\Gamma(x / 2 a)}{(2 a)^{1 / 2} \Gamma((x+a) / 2 a)}\right]^{p} .
$$

The following proof follows closely that given by Mayer in the paper to which we referred above. All real functions occurring in this paper will be assumed to be defined for all positive values of the independent variable unless otherwise specified. A function $f(x)$ is said to be convex for all $x>K$ if for every pair of values of $x$, say $x_{1}$ and $x_{2}$, for which $f(x)$ is defined, and $x_{1}>K, x_{2}>K$,

$$
f\left(\frac{x_{1}+x_{2}}{2}\right) \leqq \frac{1}{2}\left[f\left(x_{1}\right)+f\left(x_{2}\right)\right]
$$

That $f(x)$ satisfies the functional equation (1) follows from the fundamental equation for the Gamma function, $\Gamma(x+1)=x \Gamma(x)$.

We shall now show that $F(x)$ is convex. From (2) it follows ${ }^{3}$ that

$$
\frac{F^{\prime}(x)}{F(x)}=-a p \sum_{v=0}^{\infty} \frac{1}{(2 a v+x)(2 a v+a+x)} .
$$

Since $a>0, p>0$, it follows that $F^{\prime}(x) / F(x)$ is increasing with increasing $x . \log F(x)$ is therefore a convex function, and therefore $F(x)$, for the convexity of a function follows from the convexity of its logarithm as can be easily verified.

Since $F^{\prime}(x)<0, F(x)$ is a decreasing function of $x$, and we see by (1) that

\footnotetext{
1 Presented to the Society, April 13, 1940.

2 A. E. Mayer, Acta Mathematica, vol. 70 (1938), pp. 57-62.

${ }^{3}$ Mayer, loc. cit.
} 


$$
x^{-p}>F^{2}(x+a)>(x+a)^{-p} .
$$

We can now prove the main part of our theorem : if $f(x)$ is a function convex for $x>K \geqq 0$, and if it satisfies (1) for all $x>0$, then $f(x) \equiv F(x)$. From (1) it follows that the quotient $Q(x)=f(x) / F(x)$ satisfies the functional equations

$$
\begin{aligned}
Q[x+(2 n-1) a] & =1 / Q(x), \\
Q[x+2 n a] & =Q(x),
\end{aligned}
$$

where $n$ is any positive integer. We must show that $Q(x) \equiv 1$. First we suppose that $Q(x)>0$ for some particular value of $x$. Then for that value of $x$ by (4), (5), and (6)

$$
\begin{aligned}
f[x+(2 k-1) a] & =\frac{F[x+(2 k-1) a]}{Q(x)}<\frac{1}{Q(x)[x+(2 k-2) a]^{p / 2}} \\
f[x+2 k a] & =Q(x) F(x+2 k a)>\frac{Q(x)}{[x+2 k a]^{p / 2}} .
\end{aligned}
$$

Making use of the convexity of $f(x)$ for $x>K$, we may write for $x+(2 n-1) a>K$,

$$
f(x+2 n a) \leqq \frac{1}{2}\{f[x+(2 n-1) a]+f[x+(2 n+1) a]\},
$$

and therefore

$$
\frac{Q(x)}{(x+2 n a)^{p / 2}}<\frac{1}{2 Q(x)}\left\{\frac{1}{[x+(2 n-2) a]^{p / 2}}+\frac{1}{(x+2 n a)^{p / 2}}\right\},
$$

or

$$
Q^{2}(x)<\frac{1}{2}\left\{\left[\frac{x+2 n a}{x+(2 n-2) a}\right]^{p / 2}+1\right\} .
$$

If $Q(x)>0$, then $Q(x+a)=1 / Q(x)>0$, and in an exactly similar way we find that

$$
Q^{2}(x+a)=\frac{1}{Q^{2}(x)}<\frac{1}{2}\left\{\left[\frac{x+(2 n+1) a}{x+(2 n-1) a}\right]^{p / 2}+1\right\} .
$$

Since the last two displayed equations are true for all values of $n$ such that $x+(2 n-1) a>K, Q^{2}(x)=1$, and therefore $|f(x)|=F(x)$. If we assume that there exists an $x$ for which $Q(x)<0$, an argument similar to the preceding one shows again that $|f(x)|=F(x)$. Hence we conclude that for all values of $x>0|f(x)| \equiv F(x)$. We thus see, that, for every value of $x, f(x)$ is equal to either plus or minus $F(x)$. If $f(x)$ were 
equal to minus $F(x)$ for some particular value of $x$, say $x=x_{0}$, then by (1) $f\left(x_{0}+n a\right)<0$ for all $n$. Hence there would exist an $x$, say $x_{1}$, larger than $K$ such that $f\left(x_{1}\right)<0$, and $f\left(x_{1}\right)=-F\left(x_{1}\right)$. Then for every $h>0$

$$
f\left(x_{1}+h\right) \leqq \frac{1}{2}\left[f\left(x_{1}\right)+f\left(x_{1}+2 h\right)\right] \leqq \frac{1}{2}\left[-F\left(x_{1}\right)+F\left(x_{1}+2 h\right)\right] .
$$

But $F(x)$ is decreasing, and therefore $f\left(x_{1}+h\right)$ would be negative for all $h>0$, and equal to $-F\left(x_{1}+h\right)$. This function is, however, not convex. Hence $f(x)$ can never be negative, and must be identical with $F(x)$.

A function is said to be concave for $x>K$ if it satisfies equation (3) with the inequality sign reversed. It is obvious that the only function which is concave for $x>K \geqq 0$ and satisfies the functional equation (1) is $-F(x)$.

If the condition of convexity be replaced by requirement that $f(x)$ be monotone for $x>K \geqq 0$, we can obtain the following result:

THEOREM 2. The only function $f(x)$ which is monotone, not increasing, for all $x>K \geqq 0$, and satisfies the functional equation (1) is $F(x)$.

The proof of this theorem is analogous to that of Theorem 1, with the convexity condition (7) replaced by the monotone relation

$$
f(x+2 n a) \leqq f[x+(2 n-1) a] \leqq f[x+(2 n-2) a]
$$

( $n$ sufficiently large). If $x>0$ is such that $Q(x)>0$, then from (8) it follows that $Q^{2}(x)=1$. And if it is supposed that $Q(x)<0$ for some $x$, (8) leads to the contradictory inequalities $Q^{2}(x)>1, Q^{2}(x)<1$. Hence $f(x) \equiv F(x)$. The details of this proof are left to the reader.

It is also obvious from the last theorem that the only function $f(x)$ which is monotone, not decreasing, for all $x>K \geqq 0$, and satisfies (1) is $-F(x)$.

College of St. Thomas 